\title{
VISITANTES FLORAIS DE Inga edulis (FABACEAE- MIMOSOIDEAE), NA REGIÃO DO PANTANAL- PASSO DO LONTRA.
}

\section{FLORAL VISITORS OF Inga edulis (FABACEAE- MIMOSOIDEAE), IN REGION PANTANAL- PASSO DO LONTRA.}

\author{
${ }^{1}$ GONÇALVES, C.B.S.; ${ }^{2}$ SILVA, C.B.; ${ }^{3}$ CÂNDIDO, A.C.S. \\ 1- Mestre em Entomologia e conservação da biodiversidade- UFGD \\ 2- Doutorado em Ciências Farmacêuticas-UFPR \\ 3- Doutorado em Produção Vegetal- UFGD \\ REC: $10 / 09$ AC: $11 / 09$
}

\begin{abstract}
RESUMO
O objetivo deste trabalho foi investigar os visitantes florais de Inga edulis e determinar seus potenciais polinizadores. Dois indivíduos foram observados no mês de outubro de 2006, sendo realizadas observações em 12 classes de horários, correspondendo a 60 horas. As flores de 1 . edulis são vistosas e perfumadas, de antese diurna e nectaríferas, e apresentam características de espécies entomófilas. As anteras possuem pólen com $97 \%$ de viabilidade na pré-antese, $97 \%$ na antese e $98 \%$ na pós-antese. Os insetos visitantes florais de Inga edulis são de hábito diurno e não apresentaram a mesma regularidade nas visitas. Foram identificadas 20 espécies, pertencentes a 13 famílias e 5 ordens. Dentre as espécies identificadas, o maior número de representantes pertencem a família Formicidae (280), seguida por Apidae (131). Os visitantes da ordem himenóptera, principalmente formigas $(96,7 \%)$ e abelhas $(34,8 \%)$, tiveram maior pico de visitas no período matutino, entre $8 \mathrm{~h} 00$ e $10 \mathrm{~h} 00$. As abelhas Trigona sp. $(7,6)$ e A. mellifera $(22,7 \%)$ visitaram as flores pela manhã com pico nos intervalos de $7 \mathrm{~h} 00-8 \mathrm{~h} 00$ e $8 \mathrm{~h} 00-9 \mathrm{~h} 00$, respectivamente, sendo que Melipona sp. $(4,3 \%)$ concentrou suas visitas em duas classes de horários entre 9h00$10 \mathrm{~h} 00$ e $15 \mathrm{~h} 00-16 \mathrm{~h} 00$.

Palavra- chave- Ingá, polinização, abelhas visitantes, cerrado.
\end{abstract}

\begin{abstract}
.
The aim of this work was to investigate the floral visitors of Inga edulis and identify potential pollinators. Two plant individuals were observed in October 2006, being made observations at 12 hour intervals, corresponding for a duration of 60 hours. The flowers of $I$. edulis are eye- catching, scented, nectariferous, of diurnal anthesis and present characteristics of entomophilous species. The anthers have pollen with $97 \%$ viability in pre-anthesis, $97 \%$ in anthesis and $98 \%$ in post-anthesis. Floral visitors of Inga edulis are diurnal habit and did not present the same visiting patterns. 20 species have been identified, belonging to 13 families and 5 orders. Among the species identified, the largest number of representatives belong to the family Formicidae (280), followed by Apidae (131). Visitors of the himenoptera, especially ants $(96.7 \%)$ and bees (34.8\%), had greater peaks of visitng in the morning period, between 8 a.m. and 10 a.m. The bees Trigona sp. (7.6) and A. mellifera (22.7\%) visited the flowers in the morning with peaks between $7 \mathrm{~h} 00-8: 00$ hours and 8h00-9h00 hours, respectively, while Melipona sp. $(4,3 \%)$ concentrated its visits in two hours classes between $9.00-10 \mathrm{~h} 00$ and $15 \mathrm{~h} 00-16 \mathrm{~h} 00$.
\end{abstract}

Keyboards: Ingá, Polinization, visitors bee, cerrado.

\section{INTRODUÇÃO}

A polinização representa atualmente um fator de produção fundamental na condução de muitas culturas agrícolas ao redor do mundo. Esta pode ocorrer na própria 
planta, onde o grão de pólen é transportado para o estigma da flor ou, ainda, com a transferência dos grãos de pólen da antera de uma flor para o estigma de outra flor da mesma espécie, mas de pés diferentes com intervenção de agentes polinizadores, como por exemplo, os insetos (Souza et al., 2003)

A interação entre insetos e plantas garante aos vegetais o sucesso na polinização cruzada, que constitui uma importante adaptação evolutiva das plantas, aumentando o vigor das espécies, possibilitando novas combinações de fatores hereditários e aumentando a produção (Couto e Couto, 2002)

Fabaceae é a terceira maior família dentre as Angiospermas, contando com 727 gêneros e aproximadamente 19.325 espécies. No Brasil ocorrem cerca de 200 gêneros e 1500 espécies (Lewis et al. 2005.). Os representantes do gênero são arbóreos, sendo comumente encontrados em matas ciliares.

Na medicina popular, Ingá edulis tem sido utilizada como antiinflamatória e no combate à diarréia (Silva et al., 2007). Estudos sobre o perfil químico de I. edulis revelou a presença de compostos fenólicos sendo estes o acido gálico, catequina, epicatequina, miricetina -3-ramnopiranosídeo, quercetina -3- glucopiranosídeo e quercetina -3ramnopiranosídeo (Souza et al., 2007).

O trabalho teve como objetivo conhecer os visitantes florais de Inga edulis, bem como determinar os potenciais polinizadores, para contribuir na compreensão da biologia da polinização dessa espécie e a flutuação populacional dos mesmos no período do dia.

\section{MATERIAL E MÉTODOS}

2.1. Área de estudo - As investigações foram conduzidas no mês de outubro de 2006 , na base de estudos do Pantanal (BEP) localizada na margem direita do Rio Miranda, na região do Passo do Lontra, município de Corumbá, MS, nas Coordenadas 19³4'37"S e 5700'42" W. As observações foram conduzidas em dois indivíduos, situados a uma distância de $300 \mathrm{~m}$ um do outro.

O clima da região, segundo a classificação de Köppen (1948), é Aw, com temperaturas e precipitações médias anuais de $25,5^{\circ} \mathrm{C}$ e 1182 , com média de 80 chuvas ao ano, concentrados entre os meses e Novembro a Março. A deficiência hídrica dessa região é de $330 \mathrm{~mm}$ e ocorre de fevereiro a novembro, sendo mais pronunciada nos meses de agosto a outubro. O solo da região é classificado como Planossolo Hidromórfico (Embrapa, 1999).

2.2. Biologia floral - Os aspectos da biologia floral estudados foram: determinação da antese, presença de osmóforos, receptividade do estigma e viabilidade do pólen.

A receptividade do estigma foi verificada com peróxido de hidrogênio $\left(\mathrm{H}_{2} \mathrm{O}_{2}\right)$ a $20 \mathrm{~V}$ (Almeida, 1986) e a viabilidade do pólen foram estimadas pela contagem de grãos corados por carmim acético (Radford et al. 1974), ambos testados ao longo do período pré-antese.

A presença de glândulas secretoras de aroma (osmóforos) foi analisada mergulhando as inflorescências em solução de vermelho-neutro a 1\% por 10 minutos de acordo com a metodologia proposta por Vogel (1990). Os osmóforos diferenciam-se permanecendo corados pelo vermelho-neutro. O tipo de odor foi verificado mantendo algumas flores em sacos plásticos fechados, durante uma hora, para concentrar 
a substância odorífera. A presença de pigmentos que absorvem luz ultravioleta foi verificada colocando-se as inflorescências em uma solução de cloreto de ferro dissolvida em solução aquosa de éter sulfúrico a 1\% (Vogel, 1990).

2.3. Comportamento dos visitantes Florais - Os visitantes florais foram observados em cinco coletas, em dez classes de horários (7h-8h; 8h-9h; 9h-10h; 10h-11h; 11h-12h; 12h-13h; 13h-14h; 14h-15h; 15h-16h; 16h-17h), totalizando 60 horas de observação. Os insetos foram capturados por meio do uso de rede entomológica, e observações diretas sobre o comportamento dos visitantes nas flores foram acompanhadas da coleta de espécimes para identificação e registro fotográfico quando possível.

Para conhecer a dinâmica populacional dos insetos visitantes, foram calculados os índices de riqueza de espécies de Margalef, diversidade de Shannon-Wiener e de similaridade de Simpson pelo programa estatístico DIVERS versão 2.0.

\section{RESULTADOS E DISCUSSÃO}

3.1. Biologia Floral - Inga edulis é uma árvore frondosa de folhas pinadas, alado pecioladas, folíolos 4-6 jugo ovais ou obliquo elipticas, pubescentes, grandes, até 20 $\mathrm{cm}$ de raque alado. Os indivíduos investigados possuíam altura entre 10 a $15 \mathrm{~m}$. As inflorescências são axilares, algumas vezes terminais, agrupadas de 4 a 5 espigas nas axilas das folhas. As flores são vistosas e perfumadas, de antese diurna e nectaríferas, apresenta características de espécies entomófilas. O fruto é uma vagem cilíndrica, indeiscentes, com 20 a $100 \mathrm{~cm}$ de comprimento por 3 a $4 \mathrm{~cm}$ de diâmetro, contêm arilo (parte comestível). As sementes são pretas e brilhantes com 3,5 cm de comprimento e $2 \mathrm{~cm}$ de diâmetro.

As flores se abrem gradativamente na madrugada e permanecem por 24 horas, se não forem polinizadas. Após o período, estas murcham, caem ou secam e ficam presas na base da inflorescência. Quando as mesmas recebem poucas visitas, a queda ocorre por volta das $16 \mathrm{~h}$.

A entomofilia é comum em flores nectaríferas que exibem partes florais com cores brilhantes, como em Inga vulpina (Dutra et. al, 2009).

O período de receptividade do estigma ocorreu, predominantemente, entre às $7 \mathrm{~h}$ e $15 \mathrm{~h}$, sendo que o teste da receptividade, com a utilização do peróxido de hidrogênio indicou $33 \%$ de receptividade para as flores não abertas, e $80 \%$ para as flores totalmente abertas.

A presença de osmóforos, responsáveis pelo aroma exalado durante a fase receptiva da flor, foi observado na base de todas as pétalas e estames. Verificou-se que $I$. edulis exala um aroma levemente adocicado, sendo mais intenso depois da deiscência das anteras. Segundo Faegri e Van der Pijl (1980) esse aroma característico constitui um atrativo muito importante como fator de reconhecimento à longa distância pelos insetos.

Observou-se que os guias de néctar e as anteras absorvem a luz ultravioleta. Estas áreas de absorção têm um grande significado para aos insetos polinizadores, uma vez que podem orientá-los até a fonte de alimento (Balestieri \& Machado, 1998).

Através da análise de viabilidade de grãos de pólen, foi obtido uma porcentagem 
média de viabilidade de $97 \%$ na pré-antese, $97 \%$ na antese e $98 \%$ na pós-antese. (Tab.1).

Tabela 1. Viabilidade do pólen de Inga edulis em três fases do desenvolvimento das flores.

\begin{tabular}{llll}
\hline Estádios Florais & $\begin{array}{l}\text { Número de } \\
\text { Grãos de Pólen }\end{array}$ & $\begin{array}{l}\text { Média } \\
(\%)\end{array}$ & $\begin{array}{l}\text { CV } \\
(\%)\end{array}$ \\
\hline $\begin{array}{l}\text { Pré } \\
\text { antese }\end{array}$ & 3823 & $97 \pm 27,24$ & 23,1 \\
Antese & 8130 & $97 \pm 53,70$ & 17,5 \\
Pós-ântese & 1710 & $98 \pm 11,80$ & 12,1 \\
\hline
\end{tabular}

3.2. Visitantes Florais - Os insetos visitantes florais de I. edulis são de hábito diurno e não apresentaram a mesma regularidade nas visitas. Foram identificadas 20 espécies, pertencentes a 13 famílias e 5 ordens. Dentre as espécies identificadas (Tabela 2.) foi observado que o maior número de representantes pertencem a família Formicidae (280), seguida por Apidae (131). O grande número de representantes da família formicidae pode ter ocorrido devido a presença de nectários extraflorais, discos nectaríferos das flores que são comuns em leguminosas. Tais nectários atraem formigas que conferem defesa biótica contra herbívoros reduzindo as perdas e danos nos tecidos da planta. As interações formiga-planta, envolvendo as mimosoideae, exibem grande variação na especificidade e grau de proteção proporcionado pelas formigas (Pascal et al., 2000).

As formigas podem conferir papel de proteção à planta contra herbivoria. No entanto, formigas geralmente são visitantes indesejáveis às flores visitantes por ter potencial limitado como polinizadores, pois normalmente são de pequena dimensão, além da ausência de pêlos, o que dificulta a aderência do pólen; o que torna menos provável o efeito da polinização cruzada, além de possuírem glândulas com enzimas que podem prejudicar a longevidade e fertilidade dos grãos de pólen (Willmer et. al., 2009; Fernandez-Marin et al, 2006). Além disso, formigas podem interferir com polinizadores efetivos da planta, sendo que espécies agressivas pode dissuadir alguns visitantes florais, incluindo polinizadores legítimos (Gaume et. al, 2005; Ness 2006)

Tabela 2. Visitantes florais de Inga eulis, freqüência de visitas e atuação na polinização (no $=$ número). $(\mathrm{PP}=$ polinizador principal: posicionamento intrafloral adequado (legítimo), realiza visita entre plantas e freqüência de visita legítima, $\mathrm{PE}=$ polinizador eventual: posicionamento intrafloral adequado, eventualmente realiza visita entre plantas e freqüência de visita legítima, $\mathrm{PI}=$ pilhador: não contata os órgãos sexuais ao tomar néctar. 


\section{Visitantes (n) HYMENOPTERA \\ Apidae}

\section{A. mellifera \\ Melipona $s p$ \\ Centris $s p$ \\ Trigona $s p$ \\ Halictidae}

Pseudoghlochlora $s p$

Vespidae

Polistes sp

Formicidae

Pseudomirmex sp
Camponutus $s p$
Crematogaster $s p$
Cephalotes $s p$
Hipoponera $s p$
COLEOPTERA
Crisomelidae
Cerambicidae
Curculionidae
Carabidae
HEMIPTERA
Cicadelidae
Coreidae
Membracidae
DIPTERA
Cecidomidae
LEPIDOPTERA
Ninphalidae

\section{Frequência de visitas $n /(\%)$}

\section{Atuação na polinização}

$84(22,7)$

$16(4,3)$

$3(0,18)$

$28(7,6)$

PP

PI

PS

PI

PE/PI

$29(7,8)$

PI

$\begin{array}{ll}28(6,7) & \mathrm{PI} \\ 42(11,4) & \mathrm{PI} \\ 283(76,5) & \mathrm{PI} \\ 56(1,5) & \mathrm{PI} \\ 1(0,27) & \mathrm{PI} \\ 5(1,4) & \mathrm{PI} \\ 60(16,2) & \mathrm{PI} \\ 3(0,8) & \mathrm{PI} \\ 13(3,5) & \mathrm{PI} \\ 1(0,27) & \\ 5(1,4) & \mathrm{PI} \\ 1(0,27) & \mathrm{PI} \\ 45(12,2) & \mathrm{PI} \\ 1(0,27) & \mathrm{PI} \\ & \end{array}$

A espécie Crematogaster sp., bem como outros representantes de formicidae aparentemente, não realizaram polinização, uma vez que existem poucas evidências da aptidão de formigas à polinização, principalmente em razão do apterismo das operárias (CONCEIÇÃO et. al, 2004).

Os visitantes da ordem himenóptera, principalmente formigas $(96,7 \%)$ e abelhas $(34,8 \%)$, tiveram maior pico de visitas no período matutino, entre $8 \mathrm{h00}$ e $10 \mathrm{h00}$ (figura 1.). As abelhas Trigona sp. $(7,6)$ e $A$. mellifera $(22,7 \%)$ visitaram as flores pela manhã com pico nos intervalos de $7 \mathrm{~h} 00-8 \mathrm{~h} 00$ e $8 \mathrm{~h} 00-9 \mathrm{~h} 00$, respectivamente. Melipona sp. $(4,3 \%)$ concentrou suas visitas em duas classes de horários, sendo uma pela manhã 9h00- $10 \mathrm{~h} 00$ e 15h00- $16 \mathrm{~h} 00$ (figura1.). 


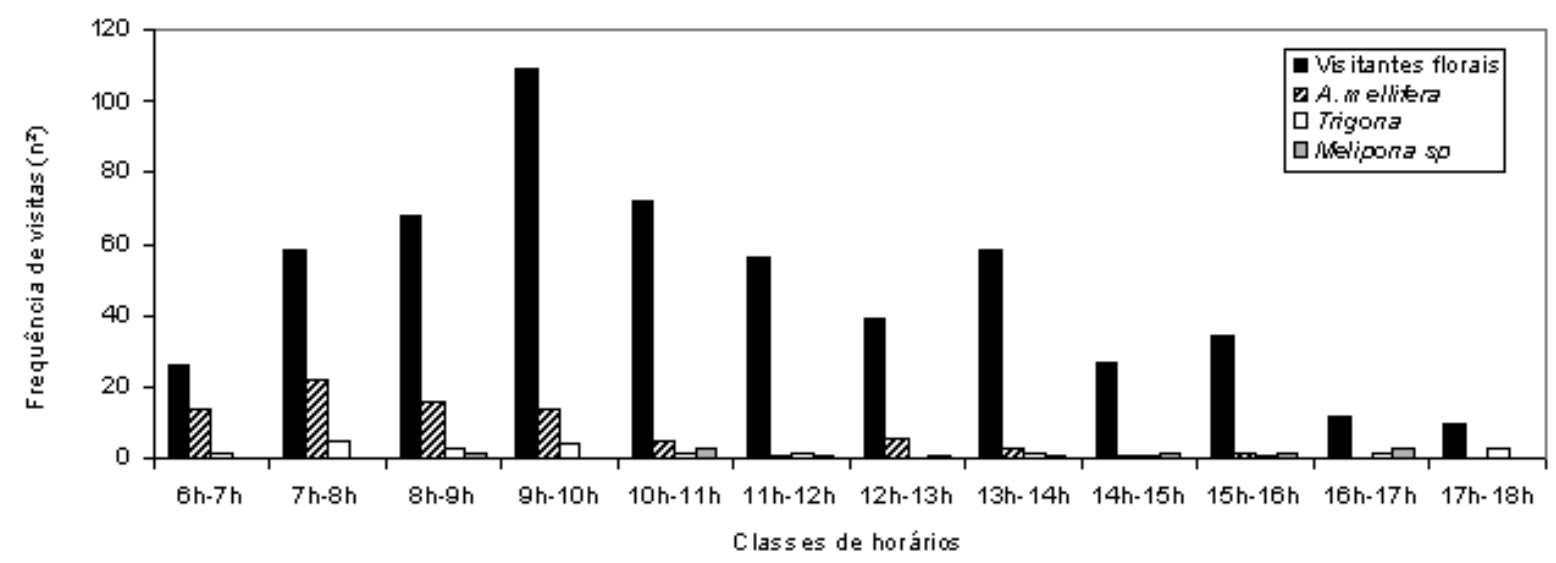

Figura 1. Freqüência de visitas registradas em flores de Inga edulis para todos os visitantes florais, bem como para as abelhas Apis mellifera,Trigona $s p$ e Melipona $s p$ em doze classes de horários.

Com relação as abelhas observadas nos dois Indivíduos de $I$. edulis, foi observado que o individuo 1 teve menor número de visitantes quando comparado ao indivíduo 2. No entanto individuo 1 foi responsável pelo maior número de visitas de $A$. mellifera, com maior freqüência no horário de $7 \mathrm{~h}-8 \mathrm{~h}$. No individuo $2 \mathrm{~A}$. mellifera e Trigon
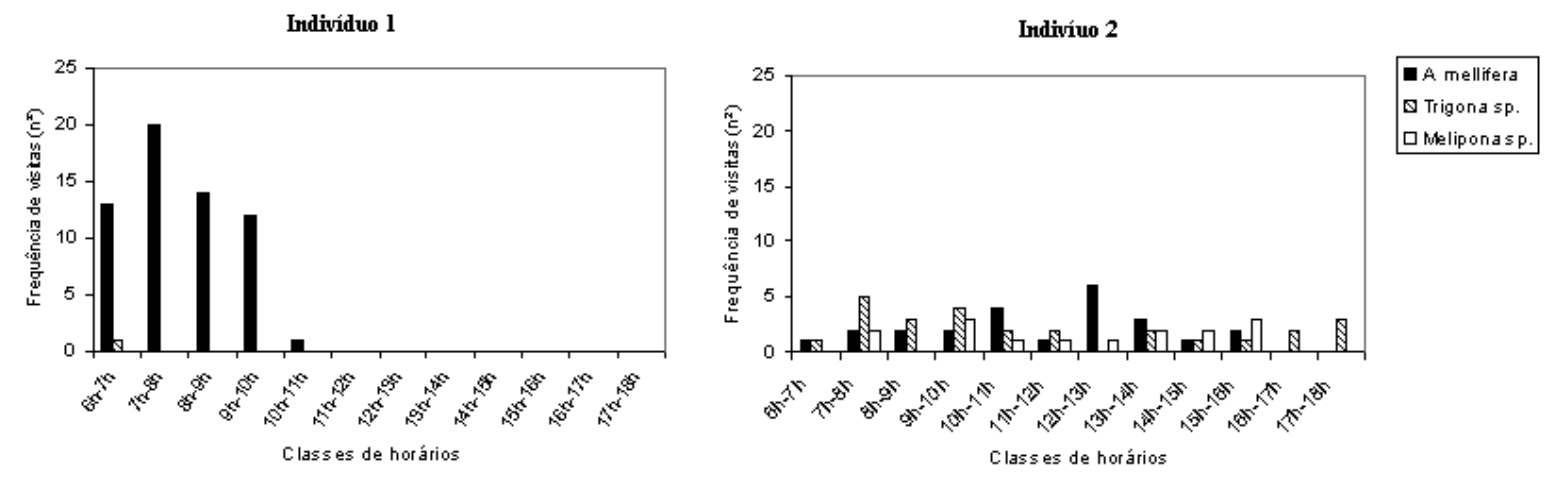

Figura 2. Freqüência de visitas registradas em flores de dois indivíuos de Inga edulis Apis mellifera, Trigona sp e Melipona $s p$ em doze classes de horários.

Apis mellifera aproxima-se da planta num vôo lento e reto dirigindo-se para flor, então pousa nas anteras contatando o estigma com a parte ventral do seu corpo e inicia a retirada do pólen com auxilio das peças bucais e dos primeiros pares de pernas, coletando em todas as anteras da flor. Em cada visita, faz pouso em várias flores, permanecendo de 5 a 10 segundos após o pouso na corola, e agarra-se aos estames desta com o primeiro par de pernas e com as outras, procura equilibrar-se sobre a flor. Em seguida, introduz a cabeça, o que the facilita o contato do aparelho bucal com o disco nectarífero situado na base do ovário. Quando a abelha deixa a 
flor, todas as peças florais que tiveram suas posições alteradas retornam à posição inicial, possivelmente realizando a polinização da flor, às vezes pousa nas pétalas onde, introduz seu aparelho bucal em direção ao nectário, não contatando o estigma. Indivíduos de $A$. mellifera apresentaram pólen na porção ventral do tórax (as duas espécies), cabeça e/ou na lateral

do corpo.

O mesmo caso ocorre com Trigona sp. e Melipona sp que em Inga edulis podem coletar pólen ou néctar. No caso de Trigona sp. e Melipona sp, uma das abelhas mais frequentes, foi observado somente coleta de néctar não contatando o estigma da flor, portanto não foi realizada a polinização.

Os representantes da família formicidae se deslocavam aleatoriamente por toda a planta, principalmente Camponotus $s p$, que visitava os nectários extraflorais, de onde coletavam o néctar. A literatura registra que vários gêneros de formigas raramente chegam até as flores e, quando o fazem limitam- se a percorrer as pétalas, sem causar-lhes danos (Conceição et al 2004). O mesmo autor ainda cita que as visitas iniciam-se tão logo as plantas emitem a inflorescências e algumas formigas mostram melhor aptidão ao carregamento de pólen podendo atuar como polinizadores casuais mas não foi o caso observado para Inga edulis. Representantes da família Lepidoptera foram observados tentando sugar o néctar das flores introduzindo o aparelho bucal, mas esta não contatava as partes reprodutoras da flor, o mesmo ocorria com Diptera e os outros insetos como o grupo de Coleoptera, que visitaram as flores em busca de néctar, mas apenas que pousavam nas folhas e alguns Crisomelideos se alimentavam das folhas (herbivoria) mas não visitavam as flores.

Os maiores índices de riqueza de Margalef $(R=2,02)$ e diversidade de Shannon $\left(H^{\prime}=1,39\right)$ foram obtidos para o individuo 1 , e maior valor para similaridade de Sorensen $(S=0,18)$ para o individuo 2 , que também apresentou uma diminuição do número das espécies e também diminuição do número de indivíduos das espécies dominantes, como mostra a Tabela 3.

Tabela. 3- Índices de riqueza, diversidade e similaridade das espécies visitantes de Inga edulis durante o período de 17 a 21 de outubro de 2005, Passo de Lontra- Corumbá /MS.

\begin{tabular}{lcc}
\hline & $\mathrm{PL} 1$ & $\mathrm{PL} 2$ \\
\hline Índice de Margalef (R1) & 2,02 & 1,33 \\
Diversidade de Shannon (H') & 1,73 & 1,39 \\
Similaridade de Sorensen (S) & 0,10 & 0,18 \\
\hline
\end{tabular}

Houve diferença para as espécies de abelhas mais frequentes entre os dois indivíduos estudados sendo estas Apis mellifera, Trigona sp e Melipona sp. A vespa Polistes sp., Cecidomidae e os demais gêneros de Formicidae foram constantes aparecendo nos dois indivíduos. Segundo Balestieri \& Machado (1998) é difícil determinar as causas que tornam algumas espécies frequentes e outra não; é provável que vários fatores influenciam, tais como fontes alternativas de alimento, competição com outros insetos e/ou baixa densidade populacional na área onde foram coletadas. 


\section{CONCLUSÕES}

As abelhas Apis mellifera e Centris sp. apresentaram comportamento de pastejo condizente com agentes polinizadores efetivos de I. edulis. As outras abelhas visitantes, devido às dimensões do corpo não contatam as anteras inferiores e o estigma e são consideradas pilhadoras

A presença de formigas nas flores de $l$. edulis, provavelmente não afeta a germinação e/ou crescimento dos tubos polínicos, devido ao fato destas não entrarem em contato direto com o pólen e/ou estígma. No entanto, elas podem influenciar a reprodução desta espécie negativamente, por dificultar o acesso dos polinizadores às estruturas reprodutivas das flores, diminuindo assim as taxas de polinização cruzada e o fluxo gênico. Estudos envolvendo múltiplas visitas por cada espécies de abelha serão necessários para identificar o potencial polinizador das demais espécies envolvidas.

\section{REFERÊNCIAS}

ALMEIDA, E. C. Biologia floral e mecanismos de reprodução em Crotaria mucrota, Revista Ceres, Viçosa, v. 33, n.190, p.528-540, 1986.

ARROYO, M.T.K. Breeding systems and polination biology in Leguminosae. In: POLHIL, R.M.; RAVEN, P.H. (Ed.) Advances in legume systematics. Kew: Royal Botanic Gardens, 1981, p.723-769.

BALESTIERI, F.C.D.M.; Machado, V.L.L. Entomofauna visitante de sibipiruna (Caesalpinia peltophoroides Benth) (Leguminosae) durante o seu período de floração.

Revista Brasileira de Entomologia, São Paulo, v. 41, n. 2-4, p. 547-554, 1998.

CONCEIÇÃO, E.S.; DELABIE, .H.C.; NETO, A. O. C. A. Entomofilia do Coqueiro em Questão: Avaliação do Transporte de Pólen por Formigas e Abelhas nas Inflorescências. Neotropical Entomology, v. 33, n. 6, p. 679-683, 2004.

COUTO, R. H. N. e COUTO, L. A. Apicultura: manejo e produtos. 2 ed. Jaboticabal: FUNEP, 2002.

DUTRA, V.F.; VIEIRA, M.F.; GARCIA, S.C.P.; LIMA, H.C. Fenologia reprodutiva, síndromes de polinização e dispersão em espécies de leguminosae dos campos rupestres do parque estadual do Itacolomi, Minas Gerais, Brasil. Rodriguésia, v. 2, p. 371-387. 2009.

EMBRAPA. Centro Nacional de Pesquisa de Solos. Sistema Brasileiro de Classificação de Solos - Brasília: Embrapa. Produção de informação; Rio de Janeiro: Embrapa solos, 1999.

FAEGRI, K.; VAN DER PIJL, L. The principles of pollination ecology. Pergamon

Press, Oxford, 1979. 
FERNANDEZ-MARIN, H., ZIMMERMAN, J.K., REHNER, S.A.; WEISLO, W.T. Active use of the metapleural glands by ants in controlling fungal infection. Proceedings of the Royal Society B: Biological Sciences, v. 273, p.1689-1695, 2006.

GAUME, L., ZACHARIAS, M. \& BORGES, R.M. Ant-plant con.icts and a novel case of castration parasitism in a myrmecophyte. Evolutionary Ecology Research, v. 7, p. 435-452, 2005.

KÖPPEN, W. Climatología com un estudio de los climas de la tierra. Ed. Fondo Cultura Económica, Cuidad de México, 1948.

LEWIS, G., SCHRIRE, B., MACKINDER, B. \& LOCK, M. Legumes of the world. Royal Botanic Gardens, Kew. 2005.

NESS, J.H. A mutualism indirect cost: the most aggressive plant bodyguards also deter pollinators. Ed. Oikos, v.113, p. 506-514, 2006.

PASCAL, L.M.; MOTTE-FLORAC, E.F.; McKEY, D.B. Secretory structures on the leaf rachis of Caesalpinieae and Mimosoideae (Leguminosae): implications for the evolution of nectary glands. American Journal of Botany, n.3, p.327-338, 2000.

RADFORD, A. E.; DICKISON, W.C.; MASSEY, J. R.; BELL, R.C. Vascular plant systematics. New York: Harper \& Row Publishers, 1974.

SILVA-PEREIRA V.; ALVES-DOS-SANTOS I.; MALAGODI-BRAGAK. S. e L. CONTRERA F. A. Forrageamento de Melissoptila thoracica Smith (Hymenoptera, Eucerini, Apoidea) em flores de Sida (Malvaceae). Revista Brasileira de Zoologia, v. 20, n. 3, p. 427-432, 2003

SILVA, E.M., SOUZA, J.N.S.; ROGEZ, H.; REES, J.F.; LARONDELLE,Y. Antioxidant activities and polyphenolic contents of fifteen selected plant species from the Amazonian region. Food Chemistry, v. 101, p. 1012-1018, 2007.

SOUZA, J.N.S, SILVA, E.M.; SILVA, M.N; ARRUDA, M.S.P.; LARONDELLE, Y.; ROGEZ, $\mathrm{H}$. Identification and Antioxidant Activity of Several Flavonoids of Inga Edulis Leaves. Journal of Brazilian Chemical Society, v. 18, n. 6, p.1276-1280, 2007.

VOGEL, S. The role of scent glands in pollination: on the structure and function of osmophores. Washington: Smithsonian Institution Libraries, 1990.

WILMER, P.G.; NUTTMAN, C.V.; RAINE, N.E.; STONE, G.N.; PATTRICK, J.G.; HENSON, K.; STILLMAN, P.; MCLLROY,L.; POTT,S.G.; KNUDSEN, J.T. Floral volatiles controlling ant behaviour. Functional Ecology, v. 23, p. 888-900, 2009. 